\title{
Jumping to conclusions in schizophrenia
}

This article was published in the following Dove Press journal:

Neuropsychiatric Disease and Treatment

I July 2015

Number of times this article has been viewed

\author{
Simon L Evans' \\ Bruno B Averbeck ${ }^{2}$ \\ Nicholas Furl ${ }^{3}$ \\ 'School of Psychology, University \\ of Sussex, Brighton, East Sussex, UK; \\ ${ }^{2}$ Laboratory of Neuropsychology, \\ National Institute of Mental Health, \\ National Institutes of Health, \\ Bethesda, MD, USA; ${ }^{3}$ Department of \\ Psychology, Royal Holloway, University \\ of London, Egham, Surrey, UK
}

Correspondence: Simon L Evans School of Psychology, University of Sussex, Brighton, East Sussex BNI 9QG, UK

Tel +44 I 273876776

Email sle27@sussex.ac.uk
Abstract: Schizophrenia is a mental disorder associated with a variety of symptoms, including hallucinations, delusions, social withdrawal, and cognitive dysfunction. Impairments on decision-making tasks are routinely reported: evidence points to a particular deficit in learning from and revising behavior following feedback. In addition, patients tend to make hasty decisions when probabilistic judgments are required. This is known as "jumping to conclusions" (JTC) and has typically been demonstrated by presenting participants with colored beads drawn from one of two "urns" until they claim to be sure which urn the beads are being drawn from (the proportions of colors vary in each urn). Patients tend to make early decisions on this task, and there is evidence to suggest that a hasty decision-making style might be linked to delusion formation and thus be of clinical relevance. Various accounts have been proposed regarding what underlies this behavior. In this review, we briefly introduce the disorder and the decisionmaking deficits associated with it. We then explore the evidence for each account of JTC in the context of a wider decision-making deficit and then go on to summarize work exploring JTC in healthy controls using pharmacological manipulations and functional imaging. Finally, we assess whether JTC might have a role in therapy.

Keywords: ketamine, decision making, delusions, fMRI, urn task

\section{Introduction to Schizophrenia}

Schizophrenia (SZ) is a mental disorder that, across the life span, affects approximately $0.3 \%-0.7 \%$ of the population. Formal diagnosis of the disorder (according to Diagnostic and Statistical Manual of Mental Disorders, Fifth Edition [DSM-V] criteria) requires symptoms to be present over an extended period of time, with so-called "positive" symptoms defined as those that represent a change in behavior or thoughts. These include delusions, hallucinations, and disorganized speech and behavior. "Negative" symptoms are those that represent a withdrawal or lack of function, and these include social withdrawal, affective flattening, anhedonia, and cognitive dysfunction. Some degree of social or occupational dysfunction must also be present. ${ }^{1}$ Typically, the onset of symptoms is observed in young adulthood. ${ }^{2}$ Some $80 \%-90 \%$ of patients with SZ experience symptomology before being formally diagnosed, reporting changes in perception, beliefs, cognition, mood, affect, and behavior. ${ }^{3}$ This is known as the prodromal (preonset) phase of the illness and can last from several weeks to several years; this phase is also referred to as an At-Risk Mental State (ARMS). ${ }^{4}$ During the prodrome, individuals typically first experience nonspecific clinical symptoms, such as depression, anxiety, or social isolation, followed by episodes of attenuated psychotic episodes: these are subpsychotic in that they are of low frequency, duration, and intensity. ${ }^{5}$ In the latter stages of the prodromal period, individuals often report unusual thoughts that could be regarded as predelusional as well as perceptual abnormalities that could be prehallucinatory. Antipsychotic medication is the first line of treatment. Antipsychotics act to block dopamine function and can ameliorate positive symptoms 
in the majority of patients. Negative symptoms are harder to treat, ${ }^{6}$ although recent evidence points to some improvement after antipsychotic therapy. ${ }^{7}$ Negative symptoms include cognitive impairment across multiple domains; this impairment tends to be fairly stable and unresponsive to treatment, although behavioral treatment might be more effective than antipsychotic therapy. ${ }^{8}$

\section{Decision-making processes in SZ}

Disorganized thoughts and speech patterns are a hallmark of SZ and exist alongside impaired motivation and a marked difficulty in maintaining and pursuing long-term life goals. ${ }^{9}$ Consequently, it is unsurprising that studies have routinely reported decision-making abnormalities in SZ patients. For example, patients have been shown to have poor accuracy on tests such as the well-used Wisconsin Card Sorting Task, which has participants learn and update card-sorting rules. ${ }^{10}$ Some studies have shown that SZ patients fail to acquire even the first sorting rule and that this is not due to a general cognitive impairment. ${ }^{11}$ Patients often show high rates of perseverative errors, suggesting a difficulty in abandoning and updating a response pattern in the face of negative feedback. ${ }^{12}$ Analyses of patient performance on the first few trials of the task (during acquisition of the first sorting rule) suggest a specific deficit in sensitivity to negative feedback, ${ }^{13}$ with patients showing a relative inability to change their behavior after receiving negative feedback. Work using other tasks, including acquisition of stimulus-response pairs ${ }^{14}$ and probabilistic learning, ${ }^{15,16}$ support this conclusion. Dopaminergic activity could represent a teaching signal that serves to modulate behavior following feedback. ${ }^{17,18}$ Dopamine pathways are implicated in SZ because atypical antipsychotics act on dopamine D2 receptors, ${ }^{19}$ and patients with $\mathrm{SZ}$ show increased striatal dopamine release under amphetamine challenge compared to healthy controls. ${ }^{20-22}$ Furthermore, recent functional magnetic resonance imaging (fMRI) work has shown that patients have reduced striatal responses during implicit learning tasks, ${ }^{23,24}$ as well as attenuated neural responses to unexpected reward. ${ }^{25}$ Thus, poor detection of environmental contingencies and a difficulty in reversal learning could be due to an unresponsive reward system driven by aberrant dopaminergic function in SZ.

Electroencephalogram (EEG) studies provide some evidence of this. A component of the event-related potential, known as the feedback negativity (FBN), is elicited by feedback informing participants that their choice has resulted in a loss. In SZ, the FBN has been shown to be diminished, suggesting that sensitivity to negative feedback is impaired; ${ }^{26}$ it is thought that the FBN is generated by the anterior cingulate cortex (ACC). ${ }^{27}$ There is evidence that the FBN could reflect the activity of dopaminergic input to motor-related neurons in ACC, ${ }^{28}$ a structure thought to be critical for error monitoring and the self-correction of behavior, ${ }^{29}$ and it has been suggested that impaired reward-based decision making is a consequence of a wider deficit in self-monitoring, since patients are less likely than controls to correct their hand movements in error trials, suggesting a self-monitoring deficit. ${ }^{30,31}$ Importantly, SZ patients show lower error signals in ACC, combined with less performance adjustment during fMRI tasks; ${ }^{32}$ structural abnormalities in prefrontal areas are often reported in imaging studies of SZ patients. ${ }^{33-36}$

The ACC and other adjacent medial prefrontal areas are thought to be critical for both self-monitoring and the attribution of mental states to other agents, an ability known as theory of mind. Theory-of-mind tasks reliably activate the medial prefrontal cortex (PFC), ${ }^{37}$ and there is some evidence that evaluation of social reward ${ }^{38}$ and social transgression $^{39}$ also engage this region. Patients have been shown to perform poorly on theory-of-mind tasks, ${ }^{40-42}$ and it has been suggested that impairments in self-monitoring and theory of mind are core deficits in SZ, giving rise to a range of positive and negative symptoms. ${ }^{42}$ Frith $^{42}$ suggests that blunted affect and social withdrawal result from difficulties in inferring the mind states of others and that experience of thought insertion and alien control (routinely reported in SZ) might stem from a failure in self-monitoring. A lack of awareness regarding one's own intentions mean that internal experiences are sometimes attributed to an external agency. Frith ${ }^{42}$ also suggests that delusional thinking (a common positive symptom of SZ, often persecutory in nature) results from faulty inferences regarding the motives of other people and there is some evidence to support this. ${ }^{43,44}$

\section{Jumping to conclusions}

Another suggestion is that delusional thinking in SZ is closely related to impaired decision-making processes. Specifically, it is suggested that delusion formation is linked to a particular pattern of performance on probabilistic decisionmaking tasks. ${ }^{45}$ Rather than, or in addition to, stemming from impaired theory of mind (where faulty inferences regarding the motives of others could lead to delusion formation), it is suggested that delusions might be due to a tendency to make inferences based on insufficient evidence. ${ }^{46}$

Some authors have further suggested that disruptions in hierarchical Bayesian inference processes might underlie both delusions and hallucinations. ${ }^{47-49}$ In this framework, 
disrupted dopamine signaling in patients with SZ leads to aberrant prediction errors. Delusions are formed by the patient to account for these prediction errors, because the prediction errors are inconsistent with reality.

Consistent with an underlying deficit in inference, it has been reliably shown that delusional symptomology is related to "jumping to conclusions (JTC)" in probabilistic reasoning tasks. Furthermore, SZ patients tend to show this pattern of behavior regardless of whether delusional symptoms are present at the time of testing. ${ }^{50}$ The paradigmatic task to demonstrate this is the so-called "urn" or "beads" task. This simple task involves two containers ("urns") containing a large number of different-colored beads in differing ratios. The participant is informed of these proportions, although the containers are hidden from view. The experimenter then presents a series of beads one at a time to the participant. After each draw, the participant can either make a guess as to which urn is being used or see another bead. Patients with SZ tend to make early decisions on this task, often making a decision after just one draw. ${ }^{51-53}$ In contrast, healthy controls tend not to make a decision before five or six draws have been completed ${ }^{54}$ and it has therefore been suggested that making a decision at two items or fewer should be classified as JTC. ${ }^{55}$

The tendency to accept hypotheses prematurely has been proposed to be critical in encouraging delusion formation by contributing to erroneous inferences ${ }^{56}$ There is considerable evidence to support the claim that JTC is linked to the presence of delusions. JTC can be detected reliably in individuals suffering from delusions, both within $\mathrm{SZ}$ and delusional disorder (a rare psychiatric condition characterized by the presence of delusions without any other symptoms). ${ }^{50} \mathrm{~A}$ JTC response style is also present in individuals at risk of delusions, those who have recovered from delusions, and more delusion-prone individuals in the general population..$^{57,58,76}$ Intriguingly, JTC can be detected in $20 \%$ of the general population: these $20 \%$ have higher levels of paranoid delusional thoughts and report more perceptual anomalies but do not differ in terms of affective (anxiety and depression) symptoms. ${ }^{59}$ Thus, JTC seems to be specifically related to delusional ideation, even in nonclinical populations. Importantly, JTC can be detected in both deluded and nondeluded SZ patients compared to healthy and psychiatric controls. ${ }^{53,60,61}$ Peters and Garety ${ }^{62}$ tested patients when actively deluded and then tested them again when in remission. The JTC bias was found to be stable, suggesting that it might exist in SZ regardless of whether delusional symptoms are present. Furthermore, successful antipsychotic treatment is not associated with a reduction in JTC. ${ }^{64}$ Other studies however contrast these findings, showing a reduction in both JTC and delusion severity following treatment initiation. ${ }^{63,65}$ Nevertheless, there is some suggestion that JTC might be a trait marker for SZ, and this is supported by the work of Van Dael et $a l^{66}$ who found evidence of a JTC response pattern in first-degree relatives of patients with SZ. There is also evidence of a JTC response style in the prodrome, where predelusional states often manifest. Broome et $\mathrm{al}^{67}$ tested individuals showing prodromal symptoms of psychosis on a modified version of the beads task with different levels of task difficulty. When the task was more difficult, the prodromal group recorded fewer draws-to-decision than the control group. Within both groups, JTC was correlated with the severity of abnormal beliefs and intolerance of uncertainty. In the prodromal group, it was also associated with impaired working memory. In contrast, healthy controls with poor working memory tended to be more conservative. A subsequent study in individuals presenting with first-episode psychosis found a higher level of JTC in this group compared to age-matched controls. ${ }^{68}$ Furthermore, in the patient group, both intelligence quotient (IQ) and delusion severity, but not working memory, were independently associated with JTC. On the basis of this evidence, JTC might constitute a risk factor in the development of psychosis.

Thus, JTC is a consistent finding in SZ, and there is strong evidence linking JTC to delusional ideation in both clinical and nonclinical groups. But why do SZ patients show a JTC response style? Various competing accounts have been proposed and we will look at each in turn.

\section{Working memory impairment}

There is some evidence to suggest that working memory might be impaired in patients who show JTC compared to those who do not. A small study by Ormrod et a ${ }^{169}$ found that visual working memory performance was affected in first-episode psychosis patients who showed a JTC response style. In addition, in SZ patients with strong current delusions, working memory (but not premorbid IQ) was worse in those who demonstrated JTC. ${ }^{70}$ As noted above, Broome et $\mathrm{a}^{67}$ found that JTC in the prodrome was linked to working memory impairments. Nevertheless, the presence of a memory aid during the "urn" task does not affect JTC in patient groups. ${ }^{51,53}$ This would appear to undermine any suggestion that JTC stems from a relative inability to maintain the task items in memory. Working memory impairments might correlate with a tendency to JTC, but the relationship is unlikely to be causal.

\section{Liberal acceptance}

One explanation for the JTC response style observed in SZ is that patients simply make decisions based on less evidence. This "liberal acceptance" account ${ }^{53,71}$ was founded on evidence 
that SZ patients tend not to converge on one particular interpretation of a situation (eg, when asked to judge the plausibility of multiple interpretations of a picture). Healthy controls ruled out interpretations that patients continued to liberally entertain, giving higher ratings to a wide range of interpretations. SZ patients are proposed to more readily accept a response option, while healthy participants are more cautious in doing so. In situations where only two (mutually exclusive) options are presented, the JTC response style manifests. To explore this, Moritz et $\mathrm{al}^{71}$ increased the number of jars to four to provide additional ambiguity: this was found to abolish the JTC in patients. Thus, JTC appears to only manifest when limited alternatives are presented. Although SZ patients made a less systematic information search and were more likely to consider less valid information, they did not inspect fewer pieces of information compared to healthy controls and thus did not demonstrate JTC. Effects were found on confidence ratings however, with patients more likely to be overconfident, using extreme confidence ratings under inappropriate circumstances. This is consistent with work showing that SZ patients are less confident of their correct answers and overconfident when they make errors, during word recall tasks. ${ }^{72,73}$

Although there were no overall differences between patients and controls, symptomatology in the patient group was linked to information search. A correlation was observed between symptom severity scores (Positive and Negative Syndrome Scale [PANSS] positive, PANSS delusion) and the degree of information search, with higher-scoring participants tending to gather less pieces of information. PANSS score did not predict use of extreme confidence ratings, but nevertheless this study would suggest that JTC can be largely abolished and can manifest as overconfidence only when information is presented in the right manner.

Liberal acceptance in patients could be underpinned by impulsiveness or motivational factors. Impulsiveness is unlikely to be a factor because patients with SZ show similar reaction times as healthy controls ${ }^{51,53}$ and draw more beads when the task is made harder..$^{51,55,74}$ Motivational factors are also unlikely to play a part: the possibility that the patients overestimate the "cost" of gathering more information (possibly due to a greater need for closure) has been discounted because patients do not seem to experience a greater cost for gathering more information. ${ }^{75}$

\section{Hypersalience of evidence}

An alternative explanation is that JTC manifests not through a lowered threshold for making a decision but through each piece of evidence being relatively "overvalued". When asked to report belief estimates after each draw, it has been shown that patients make more drastic updates after each piece of evidence. ${ }^{46,56,77}$ Speechley et $\mathrm{al}^{78}$ found that delusional patients, when asked to give likelihood ratings for each urn on each trial, gave higher ratings for whichever urn matched the current evidence, while ratings for the nonmatching urn did not differ from those of the control groups. Delusional patients were also more confident from the outset, showing a higher baseline. The authors argue that this provides evidence of a reasoning bias characterized by hypersalience of evidence that matches a hypothesis, but with reasoning that appears intact for nonmatches. The literature is inconsistent regarding patients' responses to nonmatches ("disconfirmatory" evidence). Because delusions are maintained in the face of contradictory evidence, it is unsurprising that patients tend to show a bias against disconfirmatory evidence. ${ }^{79}$ It has been argued that hypersalience could underlie this effect: hypersalience of evidence-hypothesis matches may lead to an enhancement of weak matches, leading to difficulties in integrating disambiguating information. ${ }^{80}$ Some studies point to hypersalience of disconfirmatory, as well as confirmatory, evidence: deluded patients show a tendency to overadjust when presented with potentially disconfirmatory evidence, ${ }^{53,56}$ although this effect might be linked to miscomprehension of the task, ${ }^{81}$ especially because such a tendency would contradict the evidence of reversal-learning deficits discussed in the previous section.

These findings are consistent with an aberrant salience account of SZ, ${ }^{82}$ whereby dysregulated dopamine transmission generates context-inappropriate salience attributions, potentially due to aberrant signaling in the ventral striatal dopaminergic pathway, which is thought to regulate stimulusresponse pairings. ${ }^{83}$ Moore and Sellen ${ }^{84}$ built a simple network model in which the gain, or signal-to-noise, parameter (which describes the likelihood of a node firing when presented with some input) was varied. The gain parameter was assumed to represent striatal dopaminergic activity, and increasing this parameter meant that the model successfully mimicked the JTC response style seen in research data from delusional patients. This might be overly simplistic however, because imaging studies have shown that the striatum is downregulated in $\mathrm{ARMS}^{85}$ and SZ patients. ${ }^{86}$ Nevertheless, it is important to note that evidence for hypersalience has been observed across various other cognitive biases present in $\mathrm{SZ}^{87-89}$ and as such represents a convincing account of JTC.

\section{"Self-monitoring" difficulties}

As mentioned previously, another suggestion concerning the development of delusions in SZ focuses on impairments in 
theory of mind. ${ }^{42}$ Langdon et al ${ }^{77}$ compared $35 \mathrm{SZ}$ patients with a history of delusions to healthy controls, on a battery of tasks that included two versions of the urn task, three theory-of-mind tasks, and a questionnaire on attributional biases. A JTC response style was found in the patient group, as well as impairments on the theory of mind tasks and evidence of an externalizing attributional bias. Performance on the urn tasks correlated with that of the theory-of-mind tasks, while attributional bias scores did not correlate with other task measures. Delusion proneness (measured by the questionnaire) correlated with probabilistic reasoning and theory-of-mind measures, while externalizing bias did not; IQ and memory ability were accounted for. Although it was draws-to-decision that correlated most robustly with delusion proneness, these data prompted the authors to suggest that a common underlying mechanism might operate in SZ to drive probabilistic reasoning and theory of mind deficits. They speculate that this could be a difficulty in inhibiting sensory input reflecting the immediate perceived reality, thus making patients more likely to be influenced by current data when making decisions on probabilistic reasoning tasks and making it harder to maintain an abstract viewpoint as required by theory-of-mind tasks. This echoes the hyersalience account outlined above. However, it should be noted that a large meta-analysis found no evidence for a link between theory of mind deficits and positive symptoms; instead, deficits in theory of mind were correlated with negative symptoms, disorganization, and cognitive impairment. ${ }^{90}$

There is also some suggestion that poor emotion regulation might have a role to play. In one study, 90 healthy individuals with varying levels of psychosis vulnerability (assessed by the Community Assessment of Psychic Experiences) were recruited. ${ }^{74}$ Half the sample received an anxiety induction procedure, the other half did not. Paranoid delusions and JTC were then assessed during the session. The anxiety induction procedure promoted delusions and JTC, and participants with higher psychosis vulnerability showed greater increases in paranoid delusional ideation. Furthermore, JTC appeared to mediate the association between anxiety and delusions, prompting the authors to suggest that paranoid delusions result from an interaction of anxiety and reasoning biases. In the Glöckner and Moritz ${ }^{91}$ study, it was found that increasing stress (through time pressure and the addition of affective valence) led to diminished performance in patients. Thus, improved self-monitoring in terms of better emotion regulation, combined with metacognitive training targeted at reasoning biases, could be beneficial in reducing delusion formation in SZ.

\section{Remarks}

On balance, the explanations best supported by evidence are those of liberal acceptance and hypersalience. At present, it is hard to say which is best supported by the evidence available: this is possibly because these two explanations are by no means mutually exclusive. Hypersalience and liberal acceptance could interact to generate JTC and overconfidence. One difficulty in differentiating these explanations is that the urn task offers limited information regarding learning and decision-making processes. In one of our studies, ${ }^{92}$ we investigated performance in patients on a sequence-learning task (sequences of four button presses were learnt using two buttons, feedback after each button press) and the typical urn task. Patients were able to learn the correct sequence, but learning was slower compared to that in healthy controls. Interestingly, learning from positive (but not negative) feedback in the sequence task correlated with draws-to-decision in the urn task: patients who showed a JTC response style were impaired in learning from positive feedback. Furthermore, modeling of the data revealed no evidence that patients overweigh recent evidence. Thus, these findings clearly favor a liberal acceptance account over hypersalience, but further work is needed to distinguish liberal acceptance and hypersalience, as well as to explore potential interactions with emotion regulation and other self-monitoring activities.

In the next section, we discuss work aimed at elucidating the neural basis of JTC. Various studies have attempted to induce the JTC bias in healthy controls using pharmacological manipulations; others have used fMRI.

\section{Methods of investigation Pharmacological interventions}

A drug model of JTC in healthy controls would be informative regarding the neurobiological underpinnings of JTC. There is some evidence that the noncompetitive $N$-methyl-D-aspartate (NMDA) receptor antagonist ketamine could serve as such a model. Because ketamine can be safely administered under clinical supervision and has relatively short-lived effects, the drug could represent a useful tool for studying processes underlying JTC. Ketamine infusions in healthy controls can induce behavioral and cognitive disturbances that are somewhat similar to the symptoms of SZ, ${ }^{93-95}$ infusions induce delusional thinking, ${ }^{96,97}$ and ketamine use has been linked to increased delusional symptoms in recreational users. ${ }^{98}$ Importantly, ketamine infusions in patients with SZ cause a worsening of symptoms. ${ }^{99-101}$ Ketamine has been shown to affect both glutamatergic and dopaminergic systems, ${ }^{102,103}$ potentially mimicking the aberrant dopaminergic transmission 
posited to underlie the JTC effect seen in SZ; ketamine also increases basal ganglia and thalamic activation in a manner similar to that observed in SZ patients. ${ }^{104}$

A recent study by Corlett et $\mathrm{a}^{105}$ administered ketamine to healthy controls to investigate whether faulty prediction error signals could underlie delusion formation in SZ. Prediction error is defined by the mismatch between expectations and experience, and it is probably represented by activity in the mesostriatal dopamine system. ${ }^{106}$ The prediction error signal in frontostriatal regions has been shown to correlate with delusion-like beliefs in healthy people ${ }^{107}$ and to be predictive of the severity of delusions in SZ patients. ${ }^{108}$ Disrupted prediction error signals could cause individuals to attend to and make associations with inappropriate stimuli (both internal and external), consequently developing beliefs that do not reflect real-world contingencies, ${ }^{108}$ causing a JTC style of responding. Corlett et $\mathrm{al}^{105}$ found that ketamine could strengthen the memory trace of a previously conditioned stimulus when it was presented again, without reinforcement, offering tentative support for this hypothesis. However, another study failed to find a JTC response style during the urn task in healthy controls receiving ketamine. This suggests that although ketamine can induce delusions in healthy controls, delusion formation under ketamine might not be so closely linked to JTC as in SZ, ${ }^{109}$ although possibly higher doses of ketamine might be required for JTC to manifest. Interestingly, work investigating the effects of dopamine agonists and antagonists in healthy controls has also failed to induce JTC, suggesting that straightforward modulation of the dopaminergic system might not be sufficient to reproduce the JTC response style seen in SZ. A dopamine agonist, L-dopa, had no effect on draws-to-decision, or confidence, whereas a dopamine antagonist (haloperidol) was found to reduce overconfidence but had no effect on draws-to-decision. ${ }^{110}$ Likewise, dopaminergic modulation with methamphetamine does not affect draws-todecision. ${ }^{111}$ In sum, attempts to promote JTC in controls using pharmacological interventions have been largely unsuccessful. Thus, it would seem that JTC cannot be attributed solely to general dopaminergic overactivity, at least when induced acutely: the relationship might not be linear, or perhaps chronic aberrations in dopamine firing is required for JTC to manifest. Alternatively, the disrupted interactions between several neurotransmitter systems might be critical.

\section{fMRI studies}

fMRI can potentially indicate the neural mechanisms contributing to probabilistic reasoning in the urn task. Studies in healthy controls implicate a distributed network of brain areas, including parietal cortex (typically around intraparietal sulcus), prefrontal cortex (typically dorsolateral), anterior insula, and striatum. These fMRI studies use paradigms, including draw events (stimuli for which participants choose to gather more information) and urn events (in which participants decide they have enough evidence and so select an urn). Some of these studies compared "urn task blocks" (which collapse over draw and urn decision events) with blocks where participants performed a control task on the same stimuli. The most consistent finding across these block design studies are parietal responses near the intraparietal sulcus, which are larger for urn task blocks, ${ }^{112-114}$ although some of these studies also report enhanced responses in the right dorsolateral prefrontal cortex ${ }^{113-115}$ and anterior insula. ${ }^{113,115}$ One of these studies included a SZ patient group and showed that the enhanced responses for the urn task blocks in the parietal and prefrontal cortices was reduced in these individuals, compared to the responses in healthy controls. ${ }^{113}$ This conclusion is tentative, however, as this study failed to replicate the classic JTC behavioral effect in SZ.

These block-design studies are limited, as they cannot distinguish fMRI responses to draw choice events (decisions to gather more information) from fMRI responses associated with final choices of an urn. JTC occurs when data gathering is discontinued in favor of choosing an urn, so brain areas contributing to urn choice events are likely to be involved in JTC. Studies that contrast fMRI responses to urn choices versus draw choices yield similar results as the block-design contrasts. Urn choices, compared to draw choices, activate anterior insula, striatum, ${ }^{115,116} \mathrm{ACC}$, and parietal cortex, including the intraparietal sulcus. ${ }^{116}$ A near-identical network of brain areas has also been observed in the analogous contrast in the closely related "best choice task". ${ }^{117}$ Here, this network of areas was associated with deciding on an option currently available (eg, a used car), compared to deciding to sample more possible options (eg, viewing more cars). Furl and Averbeck ${ }^{116}$ found further roles for this parietal cortex area in the urn task. Parietal cortex was more responsive during urn decisions for participants who tended to draw more and for participants who made greater adjustments to their draws-to-decision depending on prevalence of the minority bead color. These latter findings link parietal responses to individual differences in information-gathering behavior. However, this between-participant variability was within a nonclinical sample, and it remains to be confirmed whether it also extends to clinical cases such as SZ.

Involvement of areas such as striatum, dorsolateral prefrontal cortex, and intraparietal sulcus in deciding on a 
probabilistic inference rather than continued evidence seeking (as in JTC) is perhaps not surprising. These brain areas are known to contribute to related decision-making tasks. For example, measures of evidence accumulation for perceptual decisions have been associated with fMRI responses in the prefrontal cortex of psychiatrically healthy participants ${ }^{118}$ and in neural recordings from the striatum ${ }^{119}$ and intraparietal sulcus in monkeys. ${ }^{120}$ Measures of evidence accumulation for economic decisions in healthy participants are also associated with fMRI responses in dorsolateral prefrontal cortex, striatum, and intraparietal sulcus. ${ }^{121}$ Prefrontal cortex lesions are also associated with JTC. ${ }^{122}$

We hypothesize that this network of areas, which contributes to the urn task and other decision-making tasks in healthy participants, may be compromised in SZ patients who show JTC behavior. The striatum, for instance, signals errors in reward prediction and predicts successful reward learning in healthy participants ${ }^{123}$ but shows aberrant responses during reward prediction tasks in schizophrenic individuals. ${ }^{124,125}$ Aberrant prediction-related signaling in the dopaminergic striatum might also play a role in JTC behavior, as dopaminergic antipsychotic treatment abolishes JTC on an emotionally salient version of the urn task. ${ }^{64}$ In addition to the striatum, responses in the dorsolateral prefrontal cortex are also reduced in schizophrenics, compared to those in healthy individuals, when performing tasks requiring reward prediction. ${ }^{126}$ In sum, schizophrenic individuals appear to show reward-related response reductions in many of the brain areas activated by the urn task in healthy participants. Dysfunctional interactions between the dopaminergic striatum and cortical areas, such as intraparietal sulcus and dorsolateral prefrontal cortex, might explain JTC behavior in SZ patients. More brain-imaging studies using SZ patients and the urn task are required to test this hypothesis.

\section{Role in therapy}

Some studies have suggested that the JTC bias could represent an important therapeutic target. Using an emotionally salient version of the urn task, Menon et $\mathrm{al}^{64}$ found that within 2 weeks of initiating treatment with antipsychotics, patients demonstrated an increase in the number of trials-todecision alongside attenuation of psychotic symptoms and delusions. Although these measures were not correlated, baseline performance on the task had some predictive power over the individuals who would show improvements in symptomology in response to medication: JTC performance at baseline could therefore be useful in guiding treatment. Moreover, treatment-related improvements in JTC can predict probability of regaining full employment, measured over a 6-month window. This was not the case for positive symptoms or neuropsychological performance, suggesting that JTC might act independently to influence real-world functioning. ${ }^{127}$ It is important to note that most studies have failed to show improvements in JTC on the standard urn task following antipsychotic treatment, ${ }^{64,128}$ although there is some evidence to dispute this. ${ }^{63,65}$ If JTC does indeed fluctuate with delusional symptoms, it would provide strong evidence of its importance as a treatment target: interventions that specifically target cognitive biases (known as metacognitive training programs) have already been shown to improve delusions and other positive symptoms. ${ }^{129,130}$ It is also important to note that most studies typically detect JTC in only approximately $50 \%$ of their patient samples. This heterogeneity needs to be explored further, to determine how it might relate to heterogeneity of symptomatology or whether it could be an issue of task sensitivity. Again, individual differences in JTC performance could be useful in determining the best course of treatment. Training programs that aim to ameliorate the JTC response style might prove to be an important adjunct to established therapies.

\section{Conclusion}

In sum, JTC in SZ seems to be a consistent finding and there is strong evidence linking JTC to delusion formation. Both liberal acceptance and hypersalience accounts of JTC are well supported by the literature, but attempts to replicate JTC in healthy controls using pharmacological manipulations have largely failed, undermining attempts to develop a neurobiological account of JTC. fMRI studies have implicated a network involved in making urn choices, which includes striatal, insula, parietal, and prefrontal areas; further patient work is required, particularly in the context of evidence suggesting that JTC could represent a valuable therapeutic target.

\section{Disclosure}

The authors report no conflicts of interest in this work.

\section{References}

1. APA. Diagnostic and Statistical Manual of Mental Disorders. 5th ed. Washington, DC: APA; 2013.

2. van Os J, Kapur S. Schizophrenia. Lancet. 2009;374(9690):635-645.

3. Addington J. The prodromal stage of psychotic illness: observation, detection or intervention? J Psychiatry Neurosci. 2003;28(2):93-97.

4. Fusar-Poli P, Borgwardt S, Bechdolf A, et al. The psychosis high-risk state: a comprehensive state-of-the-art review. JAMA Psychiatry. 2013;70(1):107-120.

5. Schultze-Lutter F, Ruhrmann S, Berning J, Maier W, Klosterkotter J. Basic symptoms and ultrahigh risk criteria: symptom development in the initial prodromal state. Schizophr Bull. 2010;36(1):182-191. 
6. Tandon R, Keshavan MS, Nasrallah HA. Schizophrenia, "Just the Facts": what we know in 2008 part 1: overview. Schizophr Res. 2008;100(1-3):4-19.

7. Savill M, Banks C, Khanom H, Priebe S. Do negative symptoms of schizophrenia change over time? A meta-analysis of longitudinal data. Psychol Med. 2015; 45(8):1613-1627.

8. Bowie CR, Harvey PD. Cognitive deficits and functional outcome in schizophrenia. Neuropsychiatr Dis Treat. 2006;2(4):531-536.

9. Dickinson D, Bellack AS, Gold JM. Social/communication skills, cognition, and vocational functioning in schizophrenia. Schizophr Bull. 2007;33(5):1213-1220.

10. Nelson HE. A modified card sorting test sensitive to frontal lobe defects. Cortex. 1976;12(4):313-324.

11. Pantelis C, Barber FZ, Barnes TR, Nelson HE, Owen AM, Robbins TW. Comparison of set-shifting ability in patients with chronic schizophrenia and frontal lobe damage. Schizophr Res. 1999;37(3):251-270.

12. Everett J, Lavoie K, Gagnon JF, Gosselin N. Performance of patients with schizophrenia on the Wisconsin Card Sorting Test (WCST). J Psychiatry Neurosci. 2001;26(2):123-130.

13. Prentice KJ, Gold JM, Buchanan RW. The Wisconsin Card Sorting impairment in schizophrenia is evident in the first four trials. Schizophr Res. 2008;106(1):81-87.

14. Keri S, Nagy O, Kelemen O, Myers CE, Gluck MA. Dissociation between medial temporal lobe and basal ganglia memory systems in schizophrenia. Schizophr Res. 2005;77(2-3):321-328.

15. Kéri S, Kelemen O, Szekeres G, et al. Schizophrenics know more than they can tell: probabilistic classification learning in schizophrenia. Psychol Med. 2000;30(1):149-155.

16. Weickert TW, Terrazas A, Bigelow LB, et al. Habit and skill learning in schizophrenia: evidence of normal striatal processing with abnormal cortical input. Learn Mem. 2002;9(6):430-442.

17. Schultz W. Behavioral dopamine signals. Trends Neurosci. 2007;30(5):203-210.

18. Yacubian J, Glascher J, Schroeder K, Sommer T, Braus DF, Buchel C. Dissociable systems for gain- and loss-related value predictions and errors of prediction in the human brain. $J$ Neurosci. 2006;26(37):9530-9537.

19. Seeman P, Lee T, Chau-Wong M, Wong K. Antipsychotic drug doses and neuroleptic/dopamine receptors. Nature. 1976;261(5562):717-719.

20. Abi-Dargham A, Gil R, Krystal J, et al. Increased striatal dopamine transmission in schizophrenia: confirmation in a second cohort. Am J Psychiatry. 1998;155(6):761-767.

21. Laruelle M, Abi-Dargham A, van Dyck CH, et al. Single photon emission computerized tomography imaging of amphetamine-induced dopamine release in drug-free schizophrenic subjects. Proc Natl Acad Sci US A. 1996;93(17):9235-9240.

22. Breier A, Su TP, Saunders R, et al. Schizophrenia is associated with elevated amphetamine-induced synaptic dopamine concentrations: evidence from a novel positron emission tomography method. Proc Natl Acad Sci US A. 1997;94(6):2569-2574.

23. Kumari V, Gray JA, Honey GD, et al. Procedural learning in schizophrenia: a functional magnetic resonance imaging investigation. Schizophr Res. 2002;57(1):97-107.

24. Reiss JP, Campbell DW, Leslie WD, et al. Deficit in schizophrenia to recruit the striatum in implicit learning: a functional magnetic resonance imaging investigation. Schizophr Res. 2006;87(1-3):127-137.

25. Waltz JA, Schweitzer JB, Gold JM, et al. Patients with schizophrenia have a reduced neural response to both unpredictable and predictable primary reinforcers. Neuropsychopharmacology. 2009;34(6):1567-1577.

26. Morris SE, Heerey EA, Gold JM, Holroyd CB. Learning-related changes in brain activity following errors and performance feedback in schizophrenia. Schizophr Res. 2008;99(1-3):274-285.

27. Gehring WJ, Willoughby AR. The medial frontal cortex and the rapid processing of monetary gains and losses. Science. 2002;295(5563):2279-2282.

28. Holroyd CB, Coles MG. The neural basis of human error processing: reinforcement learning, dopamine, and the error-related negativity. Psychol Rev. 2002;109(4):679-709.
29. Bush G, Vogt BA, Holmes J, et al. Dorsal anterior cingulate cortex: a role in reward-based decision making. Proc Natl Acad Sci U S A. 2002;99(1):523-528.

30. Blakemore SJ, Wolpert DM, Frith CD. Abnormalities in the awareness of action. Trends Cogn Sci. 2002;6(6):237-242.

31. Fourneret P, de Vignemont F, Franck N, Slachevsky A, Dubois B, Jeannerod M. Perception of self-generated action in schizophrenia. Cogn Neuropsychiatry. 2002;7(2):139-156.

32. Carter CS, MacDonald AW 3rd, Ross LL, Stenger VA. Anterior cingulate cortex activity and impaired self-monitoring of performance in patients with schizophrenia: an event-related fMRI study. Am J Psychiatry. 2001;158(9):1423-1428.

33. Crespo-Facorro B, Kim J, Andreasen NC, O'Leary DS, Magnotta V. Regional frontal abnormalities in schizophrenia: a quantitative gray matter volume and cortical surface size study. Biol Psychiatry. 2000; 48(2):110-119.

34. Davatzikos C, Shen D, Gur RC, et al. Whole-brain morphometric study of schizophrenia revealing a spatially complex set of focal abnormalities. Arch Gen Psychiatry. 2005;62(11):1218-1227.

35. Goldstein JM, Goodman JM, Seidman LJ, et al. Cortical abnormalities in schizophrenia identified by structural magnetic resonance imaging. Arch Gen Psychiatry. 1999;56(6):537-547.

36. Pantelis C, Velakoulis D, McGorry PD, et al. Neuroanatomical abnormalities before and after onset of psychosis: a cross-sectional and longitudinal MRI comparison. Lancet. 2003;361(9354):281-288.

37. Frith U, Frith CD. Development and neurophysiology of mentalizing. Philos Trans R Soc Lond B Biol Sci. 2003;358(1431):459-473.

38. Behrens TE, Hunt LT, Woolrich MW, Rushworth MF. Associative learning of social value. Nature. 2008;456(7219):245-249.

39. Berthoz S, Armony JL, Blair RJ, Dolan RJ. An fMRI study of intentional and unintentional (embarrassing) violations of social norms. Brain. 2002;125(pt 8):1696-1708.

40. Bora E, Yucel M, Pantelis C. Theory of mind impairment in schizophrenia: meta-analysis. Schizophr Res. 2009;109(1-3):1-9.

41. Bentall RP, Rowse G, Shryane N, et al. The cognitive and affective structure of paranoid delusions: a transdiagnostic investigation of patients with schizophrenia spectrum disorders and depression. Arch Gen Psychiatry. 2009;66(3):236-247.

42. Frith CD. The Cognitive Neuropsychology of Schizophrenia. Hove: Lawrence Earlbaum Associates; 1992.

43. Mehl S, Rief W, Lullmann E, Ziegler M, Kesting ML, Lincoln TM. Are theory of mind deficits in understanding intentions of others associated with persecutory delusions? J Nerv Ment Dis. 2010;198(7):516-519.

44. Harrington L, Langdon R, Siegert RJ, McClure J. Schizophrenia, theory of mind, and persecutory delusions. Cogn Neuropsychiatry. 2005;10(2):87-104.

45. Garety PA, Freeman D. Cognitive approaches to delusions: a critical review of theories and evidence. Br J Clin Psychol. 1999;38(pt 2): 113-154.

46. Huq SF, Garety PA, Hemsley DR. Probabilistic judgements in deluded and non-deluded subjects. Q J Exp Psychol A. 1988;40(4):801-812.

47. Lee TS, Mumford D. Hierarchical Bayesian inference in the visual cortex. J Opt Soc Am A Opt Image Sci Vis. 2003;20(7):1434-1448.

48. Fletcher PC, Frith CD. Perceiving is believing: a Bayesian approach to explaining the positive symptoms of schizophrenia. Nat Rev Neurosci. 2009;10(1):48-58.

49. Dura-Bernal S, Wennekers T, Denham SL. Top-down feedback in an HMAX-like cortical model of object perception based on hierarchical Bayesian networks and belief propagation. PLoS One. 2012;7(11):e48216.

50. Fine C, Gardner M, Craigie J, Gold I. Hopping, skipping or jumping to conclusions? Clarifying the role of the JTC bias in delusions. Cogn Neuropsychiatry. 2007;12(1):46-77.

51. Dudley RE, John CH, Young AW, Over DE. Normal and abnormal reasoning in people with delusions. Br J Clin Psychol. 1997;36(pt 2): 243-258.

52. Fear CF, Healy D. Probabilistic reasoning in obsessive-compulsive and delusional disorders. Psychol Med. 1997;27(1):199-208. 
53. Moritz S, Woodward TS. Jumping to conclusions in delusional and non-delusional schizophrenic patients. Br J Clin Psychol. 2005;44(pt 2): 193-207.

54. Garety PA, Hemsley DR, Wessely S. Reasoning in deluded schizophrenic and paranoid patients. Biases in performance on a probabilistic inference task. J Nerv Ment Dis. 1991;179(4):194-201.

55. Garety PA, Freeman D, Jolley S, et al. Reasoning, emotions, and delusional conviction in psychosis. J Abnorm Psychol. 2005;114(3) 373-384.

56. Garety P. Reasoning and delusions. Br J Psychiatry. 1991;14:14-18.

57. Garety PA, Freeman D. The past and future of delusions research: from the inexplicable to the treatable. Br J Psychiatry. 2013;203(5): 327-333.

58. Ross RM, McKay R, Coltheart M, Langdon R. Jumping to conclusions about the beads task? A meta-analysis of delusional ideation and datagathering. Schizophr Bull. EPub 2015 Jan 22.

59. Freeman D, Pugh K, Garety P. Jumping to conclusions and paranoid ideation in the general population. Schizophr Res. 2008 102(1-3):254-260.

60. Menon M, Pomarol-Clotet E, McKenna PJ, McCarthy RA. Probabilistic reasoning in schizophrenia: a comparison of the performance of deluded and nondeluded schizophrenic patients and exploration of possible cognitive underpinnings. Cogn Neuropsychiatry. 2006;11(6):521-536.

61. Bentham AM, McKay AP, Quemada I, Clare L, Eastwood N, McKenna PJ. Delusions in schizophrenia: a phenomenological and psychological exploration. Cogn Neuropsychiatry. 1996;1(4):289-304.

62. Peters E, Garety P. Cognitive functioning in delusions: a longitudinal analysis. Behav Res Ther. 2006;44(4):481-514.

63. Sanford N, Woodward TS, Lecomte T, Leclerc C, Wykes T. Change in jumping to conclusions linked to change in delusions in early psychosis. Schizophr Res. 2013;147(1):207-208.

64. Menon M, Mizrahi R, Kapur S. 'Jumping to conclusions' and delusions in psychosis: relationship and response to treatment. Schizophr Res. 2008;98(1-3):225-231.

65. Woodward TS, Munz M, LeClerc C, Lecomte T. Change in delusions is associated with change in "jumping to conclusions". Psychiatry Res. 2009; 170(2-3):124-127.

66. Van Dael F, Versmissen D, Janssen I, Myin-Germeys I, van Os J, Krabbendam L. Data gathering: biased in psychosis? Schizophr Bull. 2006;32(2):341-351.

67. Broome MR, Johns LC, Valli I, et al. Delusion formation and reasoning biases in those at clinical high risk for psychosis. Br J Psychiatry Suppl. 2007;51:s38-s42.

68. Falcone MA, Murray RM, Wiffen BD, et al. Jumping to conclusions, neuropsychological functioning, and delusional beliefs in first episode psychosis. Schizophr Bull. 2015;41(2):411-418.

69. Ormrod J, Shaftoe D, Cavanagh K, et al. A pilot study exploring the contribution of working memory to "jumping to conclusions" in people with first episode psychosis. Cogn Neuropsychiatry. 2012;17(2):97-114.

70. Garety P, Joyce E, Jolley S, et al. Neuropsychological functioning and jumping to conclusions in delusions. Schizophr Res. 2013;150(2-3):570-574.

71. Moritz S, Woodward TS, Lambert M. Under what circumstances do patients with schizophrenia jump to conclusions? A liberal acceptance account. Br J Clin Psychol. 2007;46(pt 2):127-137.

72. Moritz S, Woodward TS. Memory confidence and false memories in schizophrenia. J Nerv Ment Dis. 2002;190(9):641-643.

73. Moritz S, Woodward TS. The contribution of metamemory deficits to schizophrenia. J Abnorm Psychol. 2006;115(1):15-25.

74. Lincoln TM, Lange J, Burau J, Exner C, Moritz S. The effect of state anxiety on paranoid ideation and jumping to conclusions. An experimental investigation. Schizophr Bull. 2010;36(6):1140-1148.

75. Moutoussis M, Bentall RP, El-Deredy W, Dayan P. Bayesian modelling of jumping-to-conclusions bias in delusional patients. Cogn Neuropsychiatry. 2011;16(5):422-447.

76. van der Leer L, Hartig B, Goldmanis M, McKay R. Delusion proneness and 'jumping to conclusions': relative and absolute effects. Psychol Med. 2015;45(6):1253-1262.
77. Langdon R, Ward PB, Coltheart M. Reasoning anomalies associated with delusions in schizophrenia. Schizophr Bull. 2010;36(2):321-330.

78. Speechley WJ, Whitman JC, Woodward TS. The contribution of hypersalience to the "jumping to conclusions" bias associated with delusions in schizophrenia. J Psychiatry Neurosci. 2010;35(1):7-17.

79. Woodward TS, Moritz S, Chen EY. The contribution of a cognitive bias against disconfirmatory evidence (BADE) to delusions: a study in an Asian sample with first episode schizophrenia spectrum disorders. Schizophr Res. 2006;83(2-3):297-298.

80. Speechley WJ, Ngan ET, Moritz N, Woodward TS. Impaired evidence integration and delusions in schizophrenia. J Exp Psychopathol. 2012;3(12):688-701.

81. Balzan RP, Delfabbro PH, Galletly CA, Woodward TS. Overadjustment or miscomprehension? A re-examination of the jumping to conclusions bias. Aust N Z J Psychiatry. 2012;46(6):532-540.

82. Kapur S. Psychosis as a state of aberrant salience: a framework linking biology, phenomenology, and pharmacology in schizophrenia. Am J Psychiatry. 2003;160(1):13-23.

83. Berridge KC. The debate over dopamine's role in reward: the case for incentive salience. Psychopharmacology (Berl). 2007;191(3): $391-431$.

84. Moore SC, Sellen JL. Jumping to conclusions: a network model predicts schizophrenic patients' performance on a probabilistic reasoning task. Cogn Affect Behav Neurosci. 2006;6(4):261-269.

85. Rausch F, Mier D, Eifler S, et al. Reduced activation in the ventral striatum during probabilistic decision-making in patients in an at-risk mental state. J Psychiatry Neurosci. 2015;40(1):140191.

86. Rausch F, Mier D, Eifler S, et al. Reduced activation in ventral striatum and ventral tegmental area during probabilistic decision-making in schizophrenia. Schizophr Res. 2014;156(2-3):143-149.

87. Balzan R, Delfabbro P, Galletly C, Woodward T. Reasoning heuristics across the psychosis continuum: the contribution of hypersalient evidence-hypothesis matches. Cogn Neuropsychiatry. 2012;17(5):431-450.

88. Balzan R, Delfabbro P, Galletly C, Woodward T. Confirmation biases across the psychosis continuum: the contribution of hypersalient evidence-hypothesis matches. Br J Clin Psychol. 2013;52(1): 53-69.

89. Balzan RP, Delfabbro PH, Galletly CA, Woodward TS. Illusory correlations and control across the psychosis continuum: the contribution of hypersalient evidence-hypothesis matches. J Nerv Ment Dis. 2013;201(4):319-327.

90. Ventura J, Wood RC, Hellemann GS. Symptom domains and neurocognitive functioning can help differentiate social cognitive processes in schizophrenia: a meta-analysis. Schizophr Bull. 2013;39(1):102-111.

91. Glöckner A, Moritz S. A fine-grained analysis of the jumping-toconclusions bias in schizophrenia: data-gathering, response confidence, and information integration. Judgm Decis Mak. 2009;4(7):587-600.

92. Averbeck BB, Evans S, Chouhan V, Bristow E, Shergill SS. Probabilistic learning and inference in schizophrenia. Schizophr Res. 2011;127(1-3):115-122.

93. Adler CM, Malhotra AK, Elman I, et al. Comparison of ketamineinduced thought disorder in healthy volunteers and thought disorder in schizophrenia. Am J Psychiatry. 1999;156(10):1646-1649.

94. Krystal JH, Karper LP, Seibyl JP, et al. Subanesthetic effects of the noncompetitive NMDA antagonist, ketamine, in humans. Psychotomimetic, perceptual, cognitive, and neuroendocrine responses. Arch Gen Psychiatry. 1994;51(3):199-214.

95. Malhotra AK, Pinals DA, Weingartner H, et al. NMDA receptor function and human cognition: the effects of ketamine in healthy volunteers. Neuropsychopharmacology. 1996;14(5):301-307.

96. Pomarol-Clotet E, Honey GD, Murray GK, et al. Psychological effects of ketamine in healthy volunteers. Phenomenological study. Br J Psychiatry. 2006;189:173-179.

97. Corlett PR, Honey GD, Aitken MR, et al. Frontal responses during learning predict vulnerability to the psychotogenic effects of ketamine: linking cognition, brain activity, and psychosis. Arch Gen Psychiatry. 2006;63(6):611-621. 
98. Morgan CJ, Muetzelfeldt L, Curran HV. Ketamine use, cognition and psychological wellbeing: a comparison of frequent, infrequent and ex-users with polydrug and non-using controls. Addiction. 2009;104(1):77-87.

99. Lahti AC, Holcomb HH, Medoff DR, Tamminga CA. Ketamine activates psychosis and alters limbic blood flow in schizophrenia. Neuroreport. 1995;6(6):869-872.

100. Lahti AC, WeilerMA, Tamara MichaelidisBA,ParwaniA, TammingaCA. Effects of ketamine in normal and schizophrenic volunteers. Neuropsychopharmacology. 2001;25(4):455-467.

101. Malhotra AK, Pinals DA, Adler CM, et al. Ketamine-induced exacerbation of psychotic symptoms and cognitive impairment in neuroleptic-free schizophrenics. Neuropsychopharmacology. 1997;17(3):141-150.

102. Kapur S, Seeman P. NMDA receptor antagonists ketamine and PCP have direct effects on the dopamine $\mathrm{D}(2)$ and serotonin 5-HT(2) receptors-implications for models of schizophrenia. Mol Psychiatry. 2002;7(8):837-844.

103. Moghaddam B, Adams B, Verma A, Daly D. Activation of glutamatergic neurotransmission by ketamine: a novel step in the pathway from NMDA receptor blockade to dopaminergic and cognitive disruptions associated with the prefrontal cortex. J Neurosci. 1997;17(8):2921-2927.

104. Honey GD, Corlett PR, Absalom AR, et al. Individual differences in psychotic effects of ketamine are predicted by brain function measured under placebo. J Neurosci. 2008;28(25):6295-6303.

105. Corlett PR, Cambridge V, Gardner JM, et al. Ketamine effects on memory reconsolidation favor a learning model of delusions. PLoS One. 2013;8(6):e65088.

106. Daw ND, Gershman SJ, Seymour B, Dayan P, Dolan RJ. Model-based influences on humans' choices and striatal prediction errors. Neuron. 2011;69(6):1204-1215.

107. Corlett PR, Fletcher PC. The neurobiology of schizotypy: frontostriatal prediction error signal correlates with delusion-like beliefs in healthy people. Neuropsychologia. 2012;50(14):3612-3620.

108. Corlett PR, Murray GK, Honey GD, et al. Disrupted prediction-error signal in psychosis: evidence for an associative account of delusions. Brain. 2007;130(pt 9):2387-2400.

109. Evans S, Almahdi B, Sultan P, et al. Performance on a probabilistic inference task in healthy subjects receiving ketamine compared with patients with schizophrenia. J Psychopharmacol. 2012;26(9):1211-1217.

110. Andreou C, Moritz S, Veith K, Veckenstedt R, Naber D. Dopaminergic modulation of probabilistic reasoning and overconfidence in errors: a double-blind study. Schizophr Bull. 2014;40(3):558-565.

111. Ermakova AO, Ramachandra P, Corlett PR, Fletcher PC, Murray GK. Effects of methamphetamine administration on information gathering during probabilistic reasoning in healthy humans. PLoS One. 2014;9(7):e102683.

112. Blackwood N, Ffytche D, Simmons A, Bentall R, Murray R, Howard R. The cerebellum and decision making under uncertainty. Brain Res Cogn Brain Res. 2004;20(1):46-53.

113. Krug A, Cabanis M, Pyka M, et al. Attenuated prefrontal activation during decision-making under uncertainty in schizophrenia: a multicenter fMRI study. Schizophr Res. 2014;152(1):176-183.
114. Krug A, Cabanis M, Pyka M, et al. Investigation of decision-making under uncertainty in healthy subjects: a multi-centric fMRI study. Behav Brain Res. 2014;261:89-96.

115. Esslinger C, Braun U, Schirmbeck F, et al. Activation of midbrain and ventral striatal regions implicates salience processing during a modified beads task. PLoS One. 2013;8(3):e58536.

116. Furl N, Averbeck BB. Parietal cortex and insula relate to evidence seeking relevant to reward-related decisions. J Neurosci. 2011;31(48):17572-17582.

117. Costa VD, Averbeck BB. Frontal-parietal and limbic-striatal activity underlies information sampling in the best choice problem. Cereb Cortex. 2015;25(4):972-982.

118. Ivanoff $\mathrm{J}$, Branning $\mathrm{P}$, Marois R. fMRI evidence for a dual process account of the speed-accuracy tradeoff in decision-making. PLoS One. 2008;3(7):e2635.

119. Ding L, Gold JI. Caudate encodes multiple computations for perceptual decisions. J Neurosci. 2010;30(47):15747-15759.

120. Huk AC, Shadlen MN. Neural activity in macaque parietal cortex reflects temporal integration of visual motion signals during perceptual decision making. J Neurosci. 2005;25(45):10420-10436.

121. Basten U, Biele G, Heekeren HR, Fiebach CJ. How the brain integrates costs and benefits during decision making. Proc Natl Acad Sci US A. 2010;107(50):21767-21772.

122. Lunt L, Bramham J, Morris RG, et al. Prefrontal cortex dysfunction and 'Jumping to Conclusions': bias or deficit? J Neuropsychol. 2012;6(1):65-78.

123. Roiser JP, Stephan KE, den Ouden HE, Friston KJ, Joyce EM. Adaptive and aberrant reward prediction signals in the human brain. Neuroimage. 2010;50(2):657-664.

124. Koch K, Wagner G, Schachtzabel C, et al. Neural activation and radial diffusivity in schizophrenia: combined fMRI and diffusion tensor imaging study. Br J Psychiatry. 2011;198(3):223-229.

125. Morris RW, Vercammen A, Lenroot R, et al. Disambiguating ventral striatum fMRI-related BOLD signal during reward prediction in schizophrenia. Mol Psychiatry. 2012;17(3):239-280.

126. Weickert TW, Goldberg TE, Callicott JH, et al. Neural correlates of probabilistic category learning in patients with schizophrenia. J Neurosci. 2009;29(4):1244-1254.

127. Andreou C, Treszl A, Roesch-Ely D, Kother U, Veckenstedt R, Moritz S. Investigation of the role of the jumping-to-conclusions bias for short-term functional outcome in schizophrenia. Psychiatry Res. 2014;218(3):341-347.

128. So SH, Garety PA, Peters ER, Kapur S. Do antipsychotics improve reasoning biases? A review. Psychosom Med. 2010;72(7):681-693.

129. Favrod J, Rexhaj S, Bardy S, et al. Sustained antipsychotic effect of metacognitive training in psychosis: a randomized-controlled study. Eur Psychiatry. 2014;29(5):275-281.

130. Moritz S, Veckenstedt R, Andreou C, et al. Sustained and "sleeper" effects of group metacognitive training for schizophrenia: a randomized clinical trial. JAMA Psychiatry. 2014;71(10):1103-1111.
Neuropsychiatric Disease and Treatment

\section{Publish your work in this journal}

Neuropsychiatric Disease and Treatment is an international, peerreviewed journal of clinical therapeutics and pharmacology focusing on concise rapid reporting of clinical or pre-clinical studies on a range of neuropsychiatric and neurological disorders. This journal is indexed on PubMed Central, the 'PsycINFO' database and CAS,
Dovepress

and is the official journal of The International Neuropsychiatric Association (INA). The manuscript management system is completely online and includes a very quick and fair peer-review system, which is all easy to use. Visit http://www.dovepress.com/testimonials.php to read real quotes from published authors. 